\title{
LACK OF INTERACTION BETWEEN THE DUST GRAINS AND THE ANOMALOUS RADIO JET IN THE NEARBY SPIRAL GALAXY NGC 4258
}

\author{
Seppo Laine ${ }^{1}$, Marita Krause ${ }^{2}$, Fatemeh S. Tabatabaei ${ }^{2}$, and Christos Siopis ${ }^{3}$ \\ ${ }^{1}$ Spitzer Science Center-Caltech, MS 220-6, Pasadena, CA 91125, USA; seppo@ipac.caltech.edu \\ ${ }^{2}$ Max-Planck-Institut für Radioastronomie, Auf dem Hügel 69, 53121 Bonn, Germany; mkrause@mpifr-bonn.mpg.de, tabataba@mpifr-bonn.mpg.de \\ ${ }^{3}$ Institut d'Astronomie et d'Astrophysique, Université Libre de Bruxelles, CP 226, Boulevard du Triomphe, 1050 Bruxelles, Belgium; christos.siopis@ ulb.ac.be \\ Received 2010 February 25; accepted 2010 August 14; published 2010 September 10
}

\begin{abstract}
We obtained Spitzer/IRAC 3.6-8 $\mu \mathrm{m}$ images of the nearby spiral galaxy NGC 4258 to study possible interactions between dust and the radio jet. In our analysis, we also included high-resolution radio continuum, $\mathrm{H} \alpha, \mathrm{CO}$, and X-ray data. Our data reveal that the $8 \mu \mathrm{m}$ emission, believed to originate largely from polycyclic aromatic hydrocarbon molecules and hot dust, is an excellent tracer of the normal spiral structure in NGC 4258, and hence it originates from the galactic plane. We investigated the possibility of dust destruction by the radio jet by calculating correlation coefficients between the $8 \mu \mathrm{m}$ and radio continuum emissions along the jet in two independent ways, namely, (1) from wavelet-transformed maps of the original images at different spatial scales and (2) from one-dimensional intensity cuts perpendicular to the projected path of the radio jet on the sky. No definitive sign of a correlation (or anticorrelation) was detected on relevant spatial scales with either approach, implying that any dust destruction must take place at spatial scales that are not resolved by our observations.
\end{abstract}

Key words: galaxies: individual (NGC 4258) - galaxies: ISM - galaxies: jets - galaxies: spiral - infrared: galaxies - radio continuum: galaxies

\section{INTRODUCTION}

The nearby ( $D=7.2 \mathrm{Mpc}$; Herrnstein et al. 1999) spiral galaxy NGC 4258 is known for its central black hole (Miyoshi et al. 1995; Herrnstein et al. 1998) and jets (anomalous arms), and is unique in its geometry among nearby spiral galaxies. It possesses kpc-scale anomalous radio continuum and $\mathrm{H} \alpha$ and $\mathrm{X}$-ray arms, in projection perpendicular to its normal spiral arms (Courtés \& Cruvellier 1961; van der Kruit et al. 1972) that have been shown to connect to a nuclear jet with subarcsecond resolution observations (Herrnstein et al. 1997; Cecil et al. 2000). The $\mathrm{H} \alpha$ emission along the jet is not excited by OB stars (Courtés \& Cruvellier 1961) but instead is consistent with shock excitation (e.g., Cecil et al. 1995). The anomalous radio arms emerge as linear features from the nucleus up to a projected distance of $840 \mathrm{pc}$, and then have a first bend of about $40^{\circ}$, followed by further bends, in the trailing sense relative to the galactic rotation (Krause \& Löhr 2004).

The accretion disk of the central engine has an inclination angle of $83^{\circ}$ and a position angle (p.a.) of $86^{\circ}$ (Miyoshi et al. 1995), i.e., it is oriented nearly east-west. The p.a. of the galactic disk, however, is $150^{\circ}$, which is nearly north-south, and its inclination is $72^{\circ}$ (van Albada 1980). The plane of the galactic disk and the plane of the accretion disk have significantly differing orientations, being tilted by $60^{\circ}-83^{\circ}$ with respect to each other (Krause et al. 2007). As the jets emerge perpendicular to the accretion disk, they have to travel through the galactic disk at a rather small angle. The distribution of the molecular clouds (see below) and the $\mathrm{H} \alpha$ emission of the jets indicate a direct interaction of the jets with disk material within $2 \mathrm{kpc}$ on either side of the nucleus.

Several studies (e.g., Cecil et al. 1992, 2000) have elaborated on the "jet" structure of the anomalous radio continuum arms. One of the most comprehensive studies in the radio continuum and optical wavelengths was made by Cecil et al. (2000). They find the jet to be in projection perpendicular to a nuclear accretion disk (but in space misaligned by $65^{\circ}$ ), and associated with two radio hot spots at around $1-2 \mathrm{kpc}$ from the nucleus. They find optical emission-line arcs on the leading edges of the radio hot spots. They explain the observed structure with a shock that propagates with a velocity of $350 \pm 100 \mathrm{~km} \mathrm{~s}^{-1}$ but is now stalled. The shock connects to the anomalous radio continuum arms. They interpret the overall structure with a precessing jet that has recently moved out of the plane of the galaxy. The outer anomalous arms may represent gas that has been driven out of the plane by the jet-affected halo gas that is now impacting the disk, causing an apparent curvature when projected to the plane of the sky (Wilson et al. 2001).

Single-dish ${ }^{12} \mathrm{CO} 1-0$ line observations carried out with the $30 \mathrm{~m}$ IRAM telescope revealed molecular gas in NGC 4258 only in the center and along the $\mathrm{H} \alpha$ jets, at distances up to $2 \mathrm{kpc}$ (Krause et al. 1990; Cox \& Downes 1990). It was not detected in other parts of the (central) galactic disk, down to the detection limit of about $30 \mathrm{mK}$ brightness temperature. The velocities of the molecular gas correspond primarily to the rotation of the galactic disk. Hence the molecular clouds are in the plane of the disk. High-resolution ${ }^{12} \mathrm{CO} 1-0$ observations of Krause et al. (2007) with the IRAM interferometer at Plateau de Bure revealed that the bulk of the $\mathrm{CO}$ emission comes from two parallel ridges on both sides of the linear radio jets that cross the nucleus. This morphology was confirmed by BIMA ${ }^{12} \mathrm{CO}$ $1-0$ and Sub-Millimeter Array (SMA) ${ }^{12} \mathrm{CO} 2-1$ observations (Sawada-Satoh et al. 2007). Krause et al. (2007) proposed a model in which the molecular gas temporarily accumulates along ridges surrounding the jet due to an interaction of the jet magnetic field with the molecular gas clouds.

While star formation has presumably not (yet) been triggered by the radio jet, it is of interest to study how the dust and gas have been affected by the burrowing radio jet. Previous studies of galactic jet-gas or jet-dust interactions (e.g., Whittle \& Wilson 2004) suggest that molecular gas clouds that cross the path of the jet will cause bends and knots in the jet, but eventually 

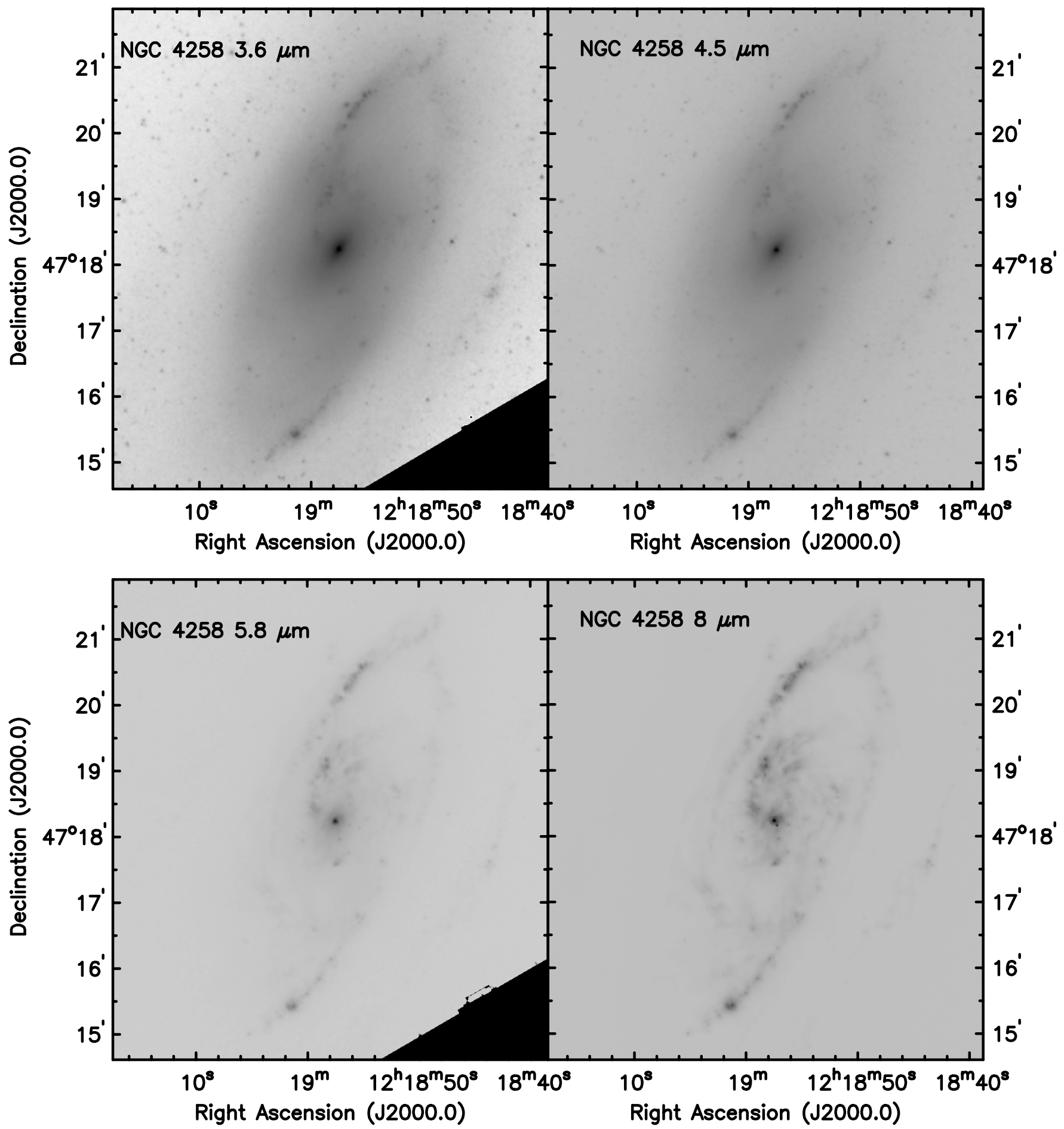

Figure 1. Gray-scale 3.6, 4.5, 5.8, and $8 \mu \mathrm{m}$ IRAC images of NGC 4258.

the jet may penetrate and ablate the cloud, in the process of ionizing and accelerating the gas. In radio galaxies, the highvelocity jets $\left(v_{\text {jet }}=10^{4}-10^{5} \mathrm{~km} \mathrm{~s}^{-1}\right)$ coming from the nuclei are expected to destroy dust grains very quickly (De Young 1998). In NGC 4258, the jet speed is an order of magnitude smaller around $1000 \mathrm{~km} \mathrm{~s}^{-1}$ (Cecil et al. 1992), and therefore it may be insufficient to cause dust destruction.

We obtained Spitzer/Infrared Array Camera (IRAC) images of NGC 4258 from 3.6 to $8 \mu \mathrm{m}$. The $8 \mu \mathrm{m}$ image is dominated by $7.7 \mu \mathrm{m}$ and $8.6 \mu \mathrm{m}$ polycyclic aromatic hydrocarbon (PAH) emission features and radiation from hot dust (e.g., Regan et al.
2004). Similarly, the $5.8 \mu \mathrm{m}$ image is dominated by the $6.2 \mu \mathrm{m}$ PAH emission feature. We compare the IRAC images to images of the radio continuum, $\mathrm{CO}, \mathrm{H} \alpha$, and $\mathrm{X}$-ray emission at a comparable resolution to investigate how the radio jet may have affected the dust properties. We use the $8 \mu \mathrm{m}$ emission as a tracer of dust emission, the $3.6 \mathrm{~cm}$ radio continuum and X-ray emission as a tracer of the jet, and we look for signs of possible dust destruction by the jet by searching for an anticorrelation between these emissions. We do this with the help of a wavelet analysis and by looking at one-dimensional intensity slices taken across the projected position of the jet in the images. 


\section{OBSERVATIONS}

NGC 4258 was observed with IRAC (Fazio et al. 2004) aboard the Spitzer Space Telescope on 2005 December 24. Observations with the channel $2(4.5 \mu \mathrm{m})$ and $4(8 \mu \mathrm{m})$ cameras were centered on the galaxy. Observations in channels $1(3.6 \mu \mathrm{m})$ and 3 $(5.8 \mu \mathrm{m})$ also covered the central part of the galaxy. The total mapped area was about $28^{\prime} \times 26^{\prime}$ in each channel. We used the small-scale cycling dithering option together with half-array map steps in the array column and row directions to cover the required area on the sky and to produce sufficient redundancy against data artifacts. We also used the high-dynamic-range observing mode, which takes a short-exposure image before a long-exposure image. The short-exposure frame times were $1.2 \mathrm{~s}$ and the long-exposure frame times were $30 \mathrm{~s}$. In this paper, we use only the $30 \mathrm{~s}$ frames, as we did not find any saturated areas in the galaxy. The pixel size in each channel is roughly $1^{\prime \prime} .2 \times 1$.'.2. The observations reported here use the S14 pipeline processing version. We ran the individual IRAC basic calibrated data (BCD) frames through the IRAC artifact mitigation software (available as contributed software on the Spitzer Web site). Subsequently we improved the muxbleed artifact mitigation in the longexposure images from channels 1 and 2 by custom software, kindly provided by J. Hora. We then made mosaics of the long-exposure frames with the Mosaicking and Point Source Extraction (MOPEX) mosaicking software (Makovoz \& Khan 2005), available at the Spitzer Science Center Web site. We corrected for the background variations in the individual frames by running the BCDs through the overlap correction algorithm first. This algorithm normalizes the spatially overlapping parts of individual BCD frames to the same mean value. The final pixel size in the mosaics is $00^{\prime \prime} 6 \times 00^{\prime \prime} 6$. MOPEX rejects outliers, such as radiation hits, during the construction of the mosaic. The standard interpolation method was used, whereby the relative overlapping areas of the input pixels from the individual BCDs are used as weights for calculating the value of the output pixel.

\section{RESULTS}

\subsection{Morphology}

The final IRAC mosaics are shown in Figure 1. The emission at $3.6 \mu \mathrm{m}$ and $4.5 \mu \mathrm{m}$ is very smooth over most of the galaxies, and traces the older stellar population in the disk. The strongest star formation regions in the spiral arms stand out from the smooth overall distribution of the emission at these wavelengths. There is likely to be a contribution from bright supergiant stars and possibly from hot dust in regions of strong recent or active star formation. The active nucleus of NGC 4258 shows up as a bright, point-like component, which is likely to have a larger contribution from hot dust as the wavelength increases from $3.6 \mu \mathrm{m}$ to $8 \mu \mathrm{m}$. The morphology becomes patchier in the 5.8 and $8 \mu \mathrm{m}$ images. At $5.8 \mu \mathrm{m}$, the stellar contribution decreases dramatically and the $6.2 \mu \mathrm{m}$ PAH and hot dust continuum components become stronger. The $8 \mu \mathrm{m}$ IRAC band still traces hot dust, but the PAH emission is likely to dominate. The hot dust and PAH emissions appear to trace the spiral arms very well (as discussed in the next section) and are found around star formation regions, but the anomalous $\mathrm{H} \alpha$ and radio continuum arms have very little hot dust or PAH emission. A similar change in morphology as the wavelength increases from $3.6 \mu \mathrm{m}$ to $8 \mu \mathrm{m}$ has been seen before, e.g., in M81 (Willner et al. 2004).

We also measured the ellipticity in the $3.6 \mu \mathrm{m}$ and $4.5 \mu \mathrm{m}$ images at radii within the major star-forming spiral arms. The ellipticity rises to a value of around $0.35-0.4$ at semimajor

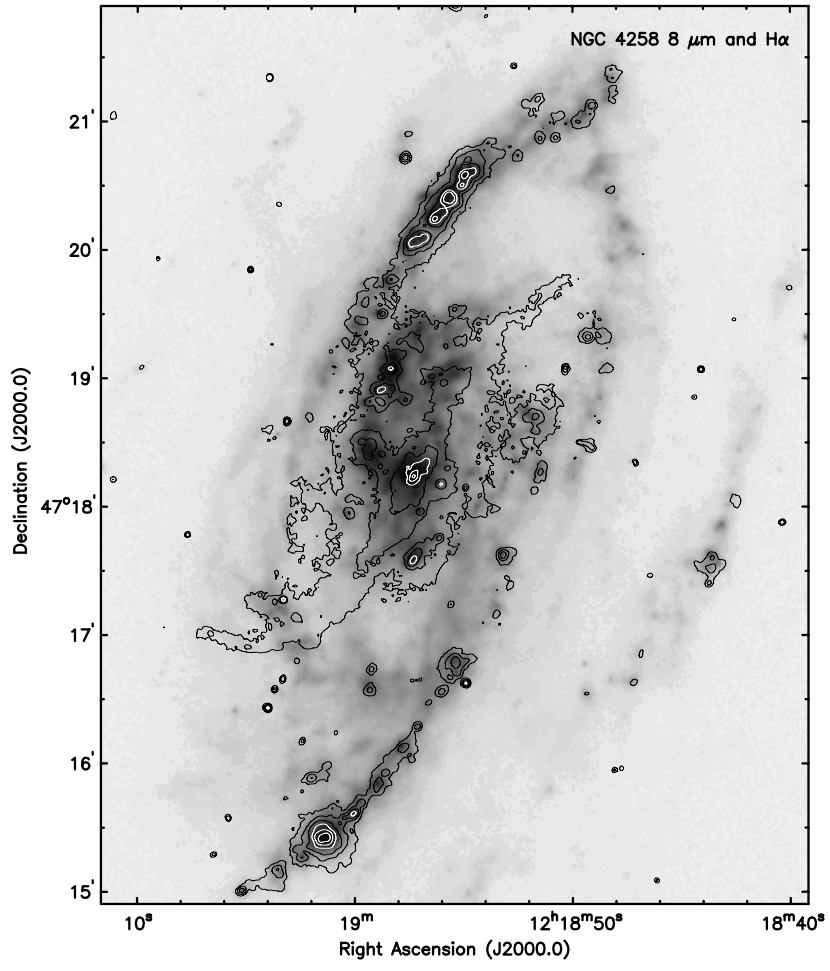

Figure 2. Gray-scale $8 \mu \mathrm{m}$ image of NGC 4258 with overlaid contours of the $\mathrm{H} \alpha$ emission from Krause et al. (2007). The contours are in arbitrary units and logarithmically spaced at $10.0 \times(2,4,8,16,32,64,128)$.

axes from $\sim 3^{\prime \prime}$ to $\sim 30^{\prime \prime}(100 \mathrm{pc}-1 \mathrm{kpc})$. The p.a. is around $146^{\circ}-148^{\circ}$. This is very similar to the $150^{\circ}$ p.a. and $72^{\circ}$ inclination of the galactic disk of NGC 4258, as determined from Hi observations by van Albada (1980). We do not see any evidence for a lense-like structure (bar) at a p.a. of $\sim 10^{\circ}$ (contrary to what was found by van Albada 1980 in $\mathrm{HI}$ ) in our images, but we cannot rule out the existence of an oval distortion either.

We do not see the radio continuum hot spots (about 50 arcsec north and 25 arcsec south of the nucleus) in the $8 \mu \mathrm{m}$ emission. They are located in relative low intensity valleys of the $8 \mu \mathrm{m}$ emission. The predicted flux densities of the hot spots, as extrapolated from the radio continuum and assuming a spectral index of 0.7 , would be less than $1 \mu \mathrm{Jy}$, well below the extended dust continuum background radiation from the disk which has fluxes about $2.5 \mathrm{mJy}$ at the locations of the radio hot spots (as integrated in $5^{\prime \prime} \times 5^{\prime \prime}$ boxes around the hot spots).

\section{2. $8 \mu \mathrm{m}$ Emission as a Tracer of Spiral Structure}

We compared the maps at $5.8 \mu \mathrm{m}$ and $8 \mu \mathrm{m}$ with various other high-resolution observations of NGC 4258 that were available to us, such as the $3.6 \mathrm{~cm}$ radio continuum (Krause \& Löhr 2004), $\mathrm{H} \alpha$ (Krause et al. 2007), X-ray (Yang et al. 2007), and ${ }^{12} \mathrm{CO} 1-0$ line observations (Krause et al. 2007). We show the most interesting overlays of $8 \mu \mathrm{m}$ with $\mathrm{H} \alpha$, and $8 \mu \mathrm{m}$ with $3.6 \mathrm{~cm}$ radio continuum in Figures 2 and 3, respectively. As Figure 2 shows, there is a very good spatial correspondence between the $8 \mu \mathrm{m}$ and $\mathrm{H} \alpha$ emission regions along the normal spiral arms of NGC 4258. However, there is much less spatial correspondence in the central part of the galaxy and along the jets. The spatial correspondence of the $8 \mu \mathrm{m}$ emission with the $3.6 \mathrm{~cm}$ radio continuum emission (Figure 3) is much weaker. They only coincide around the nucleus and along the starforming areas of the normal spiral arms. It is known that 


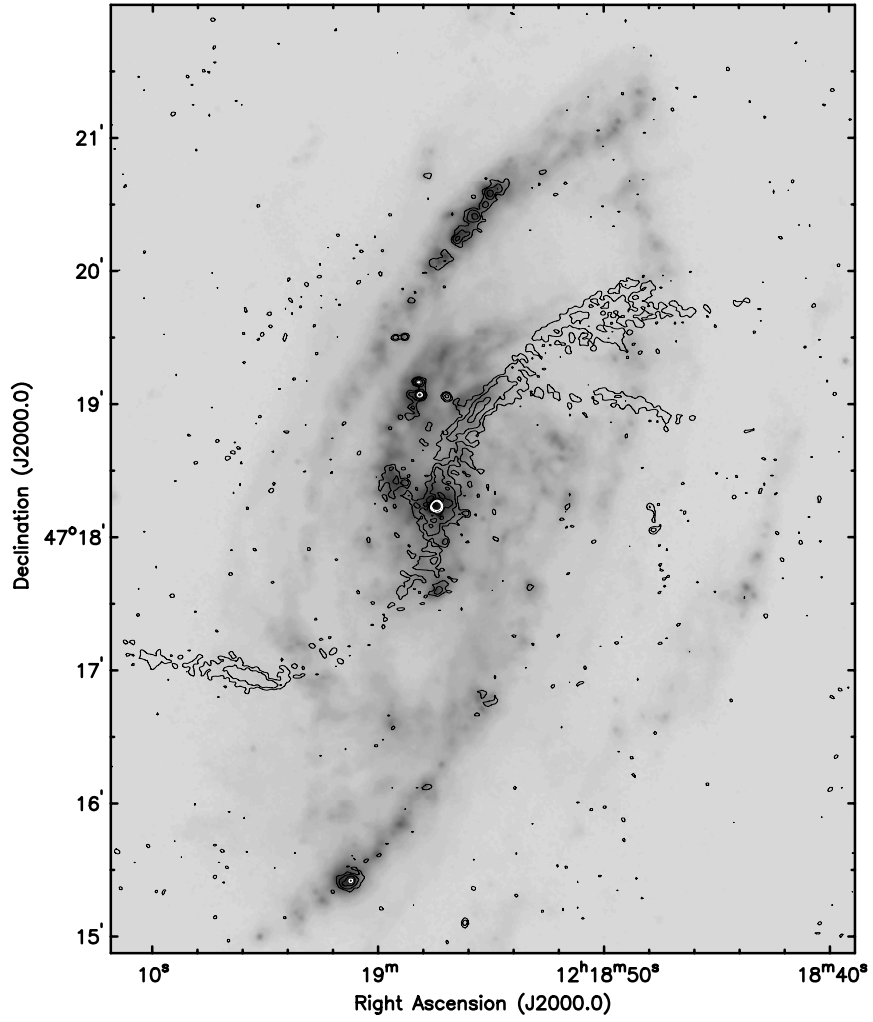

Figure 3. Gray-scale $8 \mu \mathrm{m}$ image of NGC 4258 with overlaid contours of the high-resolution radio continuum emission at $8.44 \mathrm{GHz}(\mathrm{HPBW}=2$.! $2 \times 2$ ". 4$)$ from Krause \& Löhr 2004. The contours are at $6.0 \times 10^{-6} \times(4,6,8,16,32$, 64) $\mathrm{Jy} \mathrm{beam}^{-1}$.

the radio continuum emission outside the normal star-forming spiral arms is mainly synchrotron emission from the jets (e.g., Hummel et al. 1989), whereas the $\mathrm{H} \alpha$ emission traces both the normal spiral structure and the jets (e.g., Cecil et al. 1992). The $\mathrm{X}$-ray emission morphology is similar to the radio continuum morphology, although in the central 2 arcmin the jet-like feature in the X-ray image is almost perfectly straight, as can be seen in Figure 1 of Yang et al. (2007). At larger distances the bulk of the X-ray emission follows the radio continuum very closely, although the relative intensities at various positions differ. The $\mathrm{H} \alpha$ emission along the jet follows the X-ray emission closely. It has been suggested (Wilson et al. 2001) that the jet is inclined by about $30^{\circ}$ to the disk of the galaxy and exits the disk at a distance of a few hundred parsecs from the nucleus. Beyond the nucleus the linear section of the X-ray jet could be due to shocks, triggered by the out-of-the-plane radio continuum jet, impacting the disk plane and heating the gas there. The curvature of the $\mathrm{X}$-ray and radio continuum jets beyond a few kiloparsecs from the nucleus could then be due to the lower density shock-heated gas that has been pushed out of the galactic plane (to the other side of the galactic plane with respect to the radio jet), thus causing the curvature, as seen in projection (Wilson et al. 2001).

Quite a different spatial distribution is visible in the ${ }^{12} \mathrm{CO}$ 1-0 line observations when compared to the $8 \mu \mathrm{m}$ emission (Figure 4). The $\mathrm{CO}$ emission is aligned along the jets and concentrated within the innermost $2 \mathrm{kpc}$ in radius, unlike what is seen in the $8 \mu \mathrm{m}$ dust emission. The alignment of the $\mathrm{CO}$ emission on the sides of the jet was interpreted as the molecular clouds of the disk interacting with the magnetic field of the jets (Krause et al. 2007). Such an interaction is, however, improbable for hot dust or PAHs.

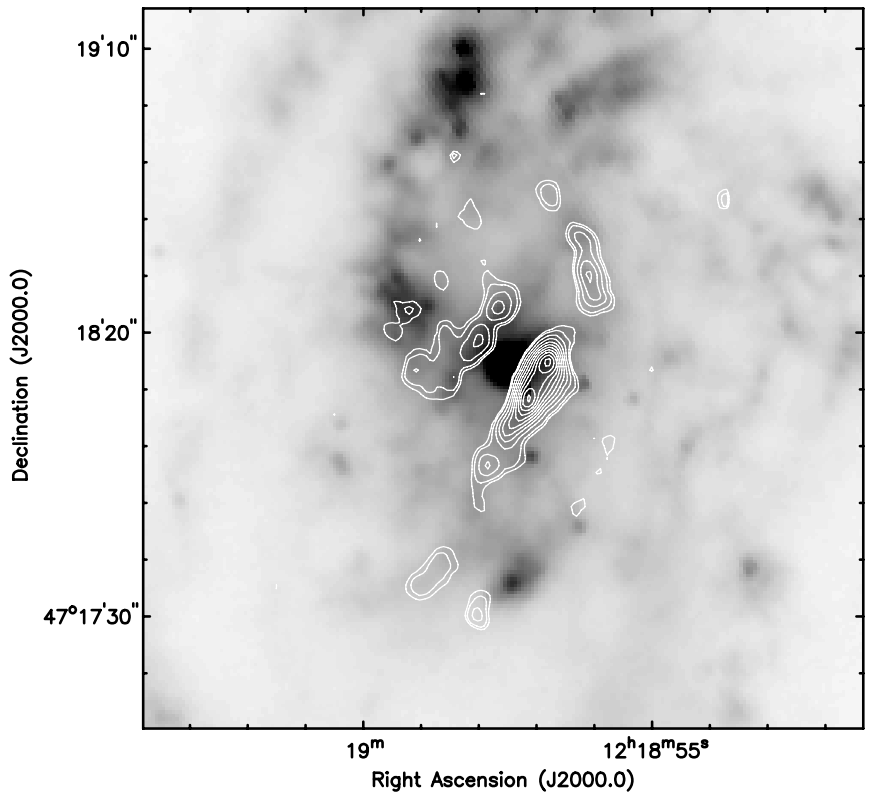

Figure 4. $\mathrm{CO}$ emission shown with contours on a gray-scale image of the $8 \mu \mathrm{m}$ emission. The contours are shown at $2.0 \times(3,4,6,8,10,12,14,16,18,20,22$, 24) $\mathrm{Jy}_{\text {beam }}{ }^{-1} \mathrm{~km} \mathrm{~s}^{-1}$. The radio jet p.a. is $-3^{\circ}$ for $r<24^{\prime \prime}$, and $-43^{\circ}$ for $r>$ $24^{\prime \prime}$, whereas the $\mathrm{CO}$ and $\mathrm{H} \alpha$ (and X-ray) emission are aligned at about $-25^{\circ}$.

We conclude that the $8 \mu \mathrm{m}$ emission, emitted mainly by PAH dust features and hot dust (which are evidently components of the galactic disk), is an excellent tracer of the normal spiral structure of NGC 4258. This is supported by the wavelet analysis of the various emissions, described in the following section.

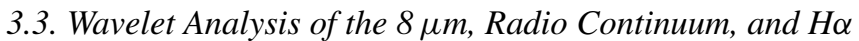 Components in NGC 4258}

To investigate the origin of the $8 \mu \mathrm{m}$ emission and its connection to the radio and $\mathrm{H} \alpha$ emissions, we compared the emitting structures on different spatial scales using a wavelet transformation. This allows us to detect systematic correlations or anticorrelations on each spatial scale.

The wavelet technique allows us to analyze the power emitted at different spatial scales (through the wavelet spectrum), and is more robust against noise than Fourier filtering (Frick et al. 2001). Cross-correlating the wavelet spectra from each wavelength lets us compare the emission structures in the various images systematically as a function of the spatial scale. We apply a two-dimensional wavelet transformation to separate the diffuse emission components from compact structures in the $8 \mu \mathrm{m}, 3.6 \mathrm{~cm}$ radio continuum, and $\mathrm{H} \alpha$ images. The wavelet coefficients of a two-dimensional continuous wavelet transformation are given by

$$
W(a, \mathbf{x})=\frac{1}{a^{\kappa}} \int_{-\infty}^{+\infty} f\left(\mathbf{x}^{\prime}\right) \psi^{*}\left(\frac{\mathbf{x}^{\prime}-\mathbf{x}}{a}\right) d \mathbf{x}^{\prime},
$$

where $\psi(\mathbf{x})$ is the analyzing wavelet, $\mathbf{x}=(x, y)$ positional coordinates, $f(\mathbf{x})$ is a two-dimensional function (the image being analyzed), and $a$ and $\kappa$ are the scale and normalization parameters, respectively, (the $*$ symbol denotes the complex conjugate). Following Frick et al. (2001) and Tabatabaei et al. (2007), we use the "Pet-Hat" wavelet to obtain a good separation of scales and to find the scale of the dominant structures in the galaxy. The "Pet-Hat" wavelet is defined in Fourier space by 


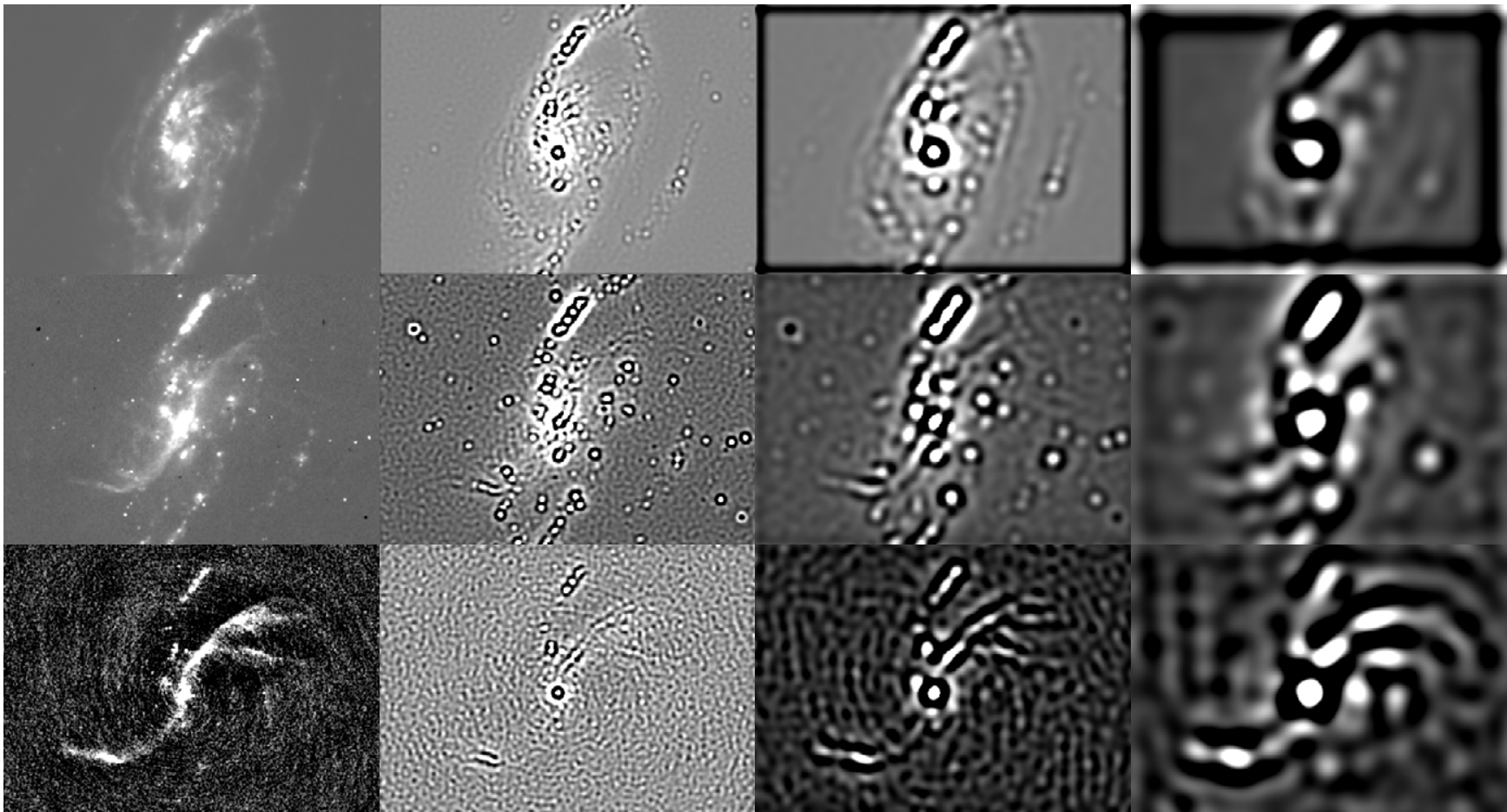

Figure 5. From top to bottom: IRAC $8 \mu \mathrm{m}, \mathrm{H} \alpha$, and $3.6 \mathrm{~cm}$ radio continuum maps at 2 '.5 resolution (first column) and their wavelet-decomposed maps on a scale of $7^{\prime \prime} .7$ (second column), 16".5 (third column), and 35".5 (fourth column). At the distance to NGC 4258, $1^{\prime \prime} \approx 35$ pc. The maps are shown in the north up, east left orientation and are centered on the nucleus of the galaxy.

the formula:

$$
\psi(\mathbf{k})= \begin{cases}\cos ^{2}\left(\frac{\pi}{2} \log _{2}\left(\frac{k}{2 \pi}\right)\right) & \pi \leqslant k \leqslant 4 \pi \\ 0 & k<\pi \text { or } k>4 \pi,\end{cases}
$$

where $\mathbf{k}$ is the wave vector and $k=|\mathbf{k}|$. The above transformation decomposes an image into "maps" of different scales. In each map, structures with the chosen spatial scale are prominent as they have larger coefficients than those with smaller or larger spatial scales.

After converting the $8 \mu \mathrm{m}, 3.6 \mathrm{~cm}$, and $\mathrm{H} \alpha$ images into the same geometry, size, and resolution (2..5), we decomposed them into 10 spatial scales ranging from $5^{\prime \prime}$ to $165^{\prime \prime 4}$ using Equations (1) and (2). Figure 5 shows the original $8 \mu \mathrm{m}$, $3.6 \mathrm{~cm}$, and $\mathrm{H} \alpha$ images together with their decomposed maps on three scales. On the scale of $a=7^{\prime \prime} .7(270 \mathrm{pc})$, the $8 \mu \mathrm{m}$ emission reveals small clumps that make up segments of rings or spiral arms, even where the $\mathrm{H} \alpha$ emission is faint or absent. Furthermore, in contrast to the $\mathrm{H} \alpha$ and radio continuum emissions, there are filamentary and flocculent structures at $8 \mu \mathrm{m}$, some of which appear more extended in the northern than in the southern half of the galaxy. The outer spiral arms are better visible on the $a=16.5$ (580 pc) scale. At this scale the strong star-forming region in the northern arm is the only common structure with the radio emission. On the next larger scale, $a=35^{\prime \prime} .5(1.2 \mathrm{kpc})$, the dominant dust-emitting structures are found in the center and around the strong star-forming region located in the northern spiral arm.

Apart from the center the radio continuum emission is dominated by the jet and the star-forming region in the northern

\footnotetext{
4 To have both physically meaningful results and a sufficiently large number of independent points, the scale $a$ varies between a minimum of about twice the resolution and a maximum of about half of the image size.
}

spiral arm on all scales. A large amount of the $\mathrm{H} \alpha$ emission appears as dispersed clumps of star-forming regions. The $\mathrm{H} \alpha$ emission jet appears as a smooth, elongated structure on scales larger than $0.5 \mathrm{kpc}$. However, there is not any trace of a jet-like structure in the $8 \mu \mathrm{m}$ wavelet-decomposed maps. The $8 \mu \mathrm{m}$ emission traces the stellar spiral arms much better than the $\mathrm{H} \alpha$ or radio continuum emissions.

To quantify any correlation or anticorrelation of the dust emission with the $\mathrm{H} \alpha$ and radio continuum emissions along the jet, we focus on the region covered by the radio jet. We made maps of the radio continuum, $\mathrm{H} \alpha$, and $8 \mu \mathrm{m}$ emissions by including only regions corresponding to the radio continuum intensities larger than a threshold (the point sources and the stellar spiral arms had been subtracted from the radio continuum map before doing this). These maps were then decomposed in the same way for the whole galaxy. In Figure 6, we plot the wavelet spectrum, i.e., the distribution of emitting power in terms of the spatial scale for each map given by

$$
M(a)=\int_{-\infty}^{+\infty} \int_{-\infty}^{+\infty}|W(a, \mathbf{x})|^{2} d \mathbf{x} .
$$

A smooth distribution of the power spectrum is expected if the emission along the masked radio jet originates from a real jet. The radio-emitting power increases toward the $20^{\prime \prime}-40^{\prime \prime}$ $(0.7-1.4 \mathrm{kpc})$ scales and decreases smoothly beyond these scales. There does not appear to be any overall dominant power at small spatial scales. However, the smallest analyzed spatial scale $\left(5^{\prime \prime}\right.$ or $\left.170 \mathrm{pc}\right)$ has the most power among the first four analyzed spatial scales at $8 \mu \mathrm{m}$. This indicates patchiness of the dust emission along the radio jet region. These emission patches probably emerge from gas and dust clouds in the disk. It is notable that all three wavelengths share the same broad peak in their wavelet power spectrum between $20^{\prime \prime}$ and $40^{\prime \prime}$. This reflects mostly the enhanced emission (and size) of the central region. 


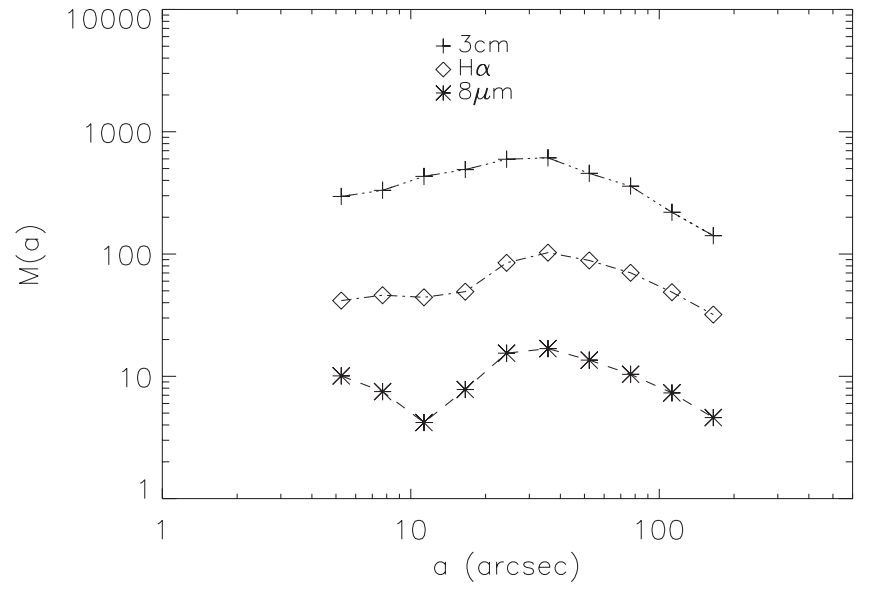

Figure 6. Wavelet power spectrum for the $8 \mu \mathrm{m}, \mathrm{H} \alpha$, and $3.6 \mathrm{~cm}$ emissions along the track covered by the radio continuum jet. The data points correspond to the scales of $5.2,7.7,11.3,16.6,24.3,35.6,52.3,67.7,112.5$, and 165.0 arcsec. The power is shown in arbitrary units.

The wavelet spectrum of the $\mathrm{H} \alpha$ emission is intermediate in nature between those of the radio and dust emissions. It is not dominated by the small-scale emission, in contrast to the $8 \mu \mathrm{m}$ emission, but it also does not increase smoothly toward the maxima at $0.7-1.4 \mathrm{kpc}$ scales (unlike the radio continuum power spectrum). H $\alpha$ shares the smooth decline of the other two power spectra past the peak. This may be a consequence of the $\mathrm{H} \alpha$ emission consisting of both emission from the $\mathrm{H} \alpha$ jet and the underlying disk structures, specifically, $\mathrm{H}$ in regions.

We calculate the correlation coefficients on each scale with

$$
r_{\mathrm{w}}(a)=\frac{\iint W_{1}(a, \mathbf{x}) W_{2}^{*}(a, \mathbf{x}) d \mathbf{x}}{\left[M_{1}(a) M_{2}(a)\right]^{1 / 2}},
$$

where the subscripts refer to two images of the same size and linear resolution. The value of $r_{\mathrm{w}}$ varies between -1 (total anticorrelation) and +1 (total correlation).

The uncertainty in $r_{\mathrm{w}}$ can be approximated by

$$
\Delta r_{\mathrm{w}}(a)=\frac{\sqrt{1-r_{w}^{2}}}{\sqrt{n-2}},
$$

where $n=2.13(L / a)^{2}$ is the number of independent points and $L$ is the size of the maps. Plotting $r_{\mathrm{w}}$ against the spatial scale shows how well structures at different scales are correlated in intensity and location. The wavelet cross-correlation coefficients $r_{w}$ are shown in Figure 7. The dust emission is not correlated with the radio emission at small scales. A significant $8 \mu \mathrm{m}-3.6 \mathrm{~cm}$ correlation $\left(r_{\mathrm{w}}>0.75 ;^{5}\right.$ although with large uncertainty), is found at large scales $\left(a>36^{\prime \prime}\right)$ due to the extended emission from the bright central part and because of the artificial smearing of the two emissions by using the same masking (at $2 \sigma$ radio continuum rms level, covering the jet) in both maps. In general, the dust emission has a better correlation with the $\mathrm{H} \alpha$ emission (even in the radio jet region) than with the radio continuum emission. The weakest correlation occurs around $a \sim 11^{\prime \prime}$ at about the width of the $\mathrm{H} \alpha$ jet. A good small spatial scale $8 \mu \mathrm{m}-\mathrm{H} \alpha$ correlation is possibly due to the starforming regions in the disk that are projected on the radio jet along the line of sight. If so, then a superposition of the emission from the jet and the underlying galaxy disk makes it very

\footnotetext{
5 A coefficient $r_{\mathrm{W}}$ of $>0.75$ means that more than $50 \%$ of the total variation in one of the variables can be explained by a linear relationship between the two variables.
}

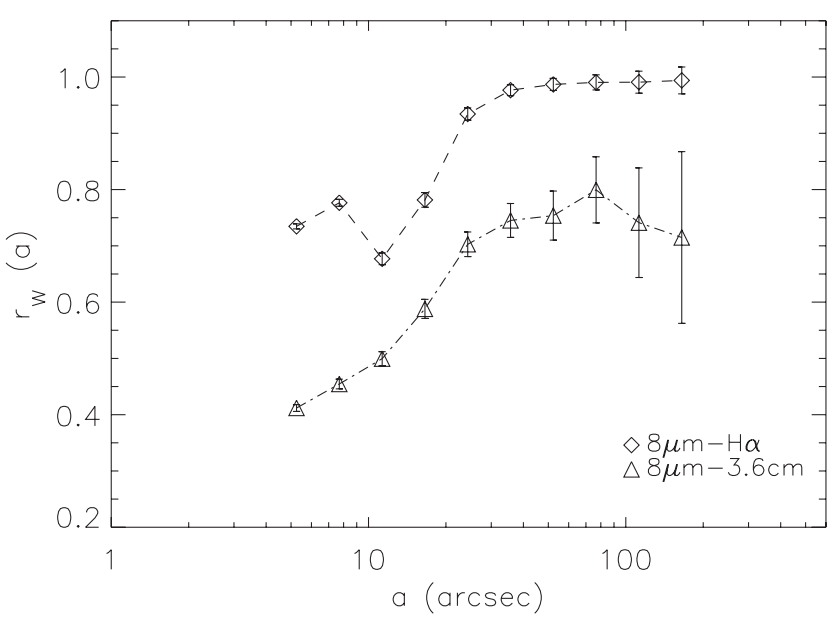

Figure 7. Wavelet cross-correlation of the $8 \mu \mathrm{m}$ emission with the $\mathrm{H} \alpha$ and $3.6 \mathrm{~cm}$ radio continuum emissions along the track covered by the radio continuum jet, as a function of spatial scale.

difficult to detect an anticorrelation between the dust and the radio emissions along the jet.

The wavelet analysis confirms that the $8 \mu \mathrm{m}$ emission traces the normal stellar spiral arms much better than the radio continuum and $\mathrm{H} \alpha$ emissions. The long continuous spiral arm in the northeast stands out, as does the split stellar spiral arm in the south and a long section of a spiral arm in the northwest. It is also clear that the emission just north (northeast) of the nucleus at declination $47^{\circ} 19^{\prime}$ comes from a spiral arm rather than from the jet. In this region, no $\mathrm{CO}$ line emission has been detected, which is consistent with the result that the $\mathrm{CO}$ emission is only found flanking the jet (see Krause et al. 1990). Even more notably, the wavelet analysis shows that the $8 \mu \mathrm{m}$ emission does not trace the radio continuum jet emission at all.

\subsection{Emission Profiles Across the Jet}

In this section, we further examine the dust to radio continuum correlation by taking one-dimensional slices in the $8 \mu \mathrm{m}$ and $3.6 \mathrm{~cm}$ radio continuum emissions perpendicular to the skyprojected orientation of the radio jet at several positions across the galaxy. The positions of the slices were chosen to sample the radio jet at various positions along its length. The slice positions are shown in Figure 8, and the emission profiles along the slices in Figure 9. The only slice in which visual inspection shows evidence of an anticorrelation between the radio continuum and $8 \mu \mathrm{m}$ emissions is at position 10 , which lies far out in the outer spiral arms, where the radio continuum jet has split into several branches. We believe that this is the result of a fortuitous distribution of the two emissions at this location, rather than of any physical PAH grain destruction by the radio jet. In several other slices, we see a phase offset between the radio continuum and $8 \mu \mathrm{m}$ emissions, but there does not appear to be any clear pattern in the magnitude of the offset, in the leading or trailing sense, as a function of the distance from the nucleus of the galaxy or the side of the galaxy (and jet). We varied the orientation and the length of the slices in a few cases and confirmed that the lack of any correlation or anticorrelation in the slices is not due to their orientation or length (see also the note below about performing the correlation coefficient calculation by excluding points at the ends of the slices).

We can quantify the preceding remarks, obtained from visual inspection, by calculating correlation coefficients between the $8 \mu \mathrm{m}$ and the radio continuum emission profiles for each slice 


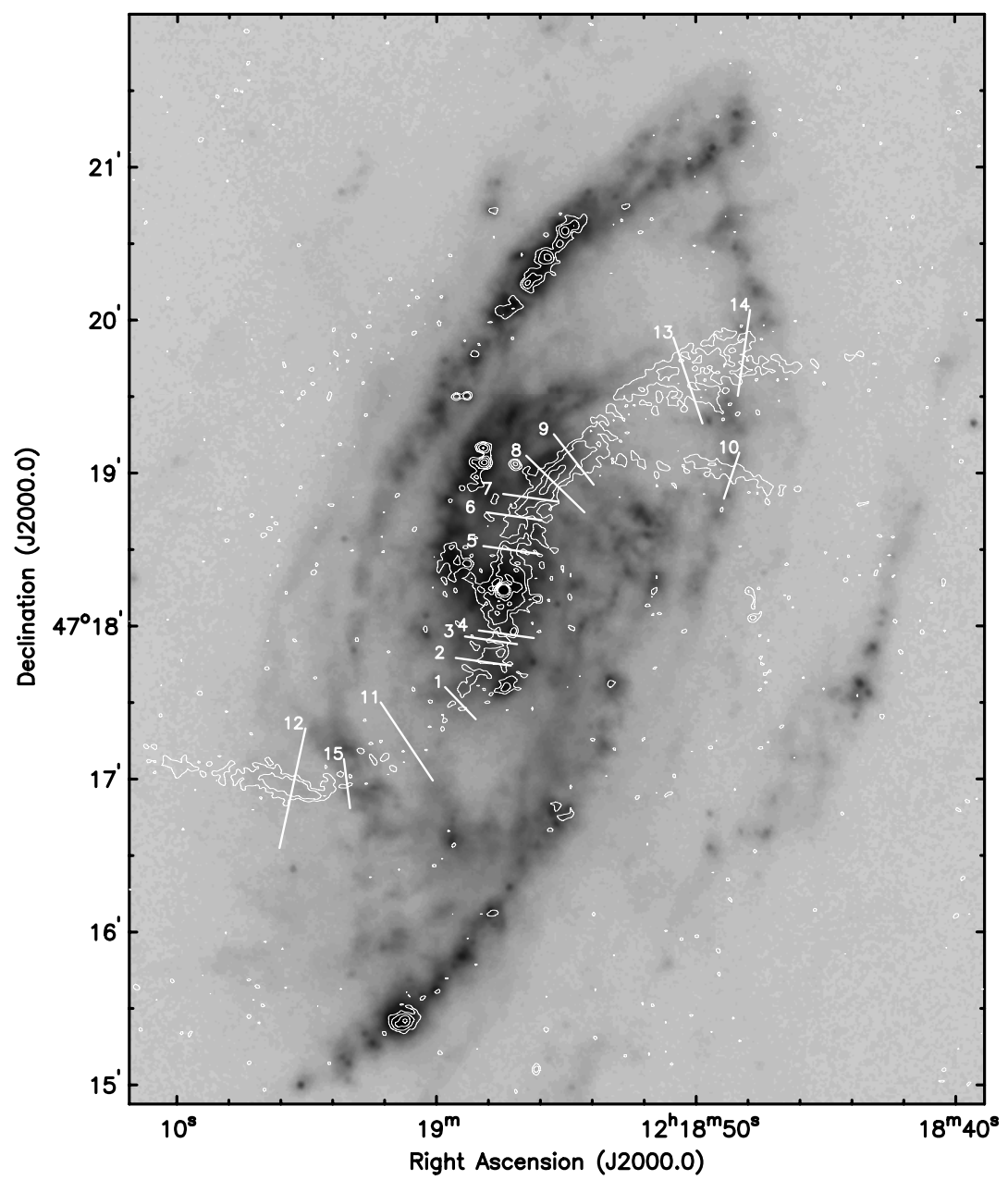

Figure 8. Same as Figure 3, but with the labeled positions of the slices taken across the radio jet and shown in Figure 9.

presented in Figure 9. We calculated the parametric Pearson (linear) correlation and the non-parametric Spearman (rankorder) correlation, along with their $P$-values, for all the slices. In principle, the Spearman correlation is more appropriate since there is no reason to expect that the emission profiles are drawn from normally distributed populations. In practice, both methods produced similar results, which are shown in Table 1 . The $P$ value is the probability of obtaining the calculated correlation coefficient assuming the null hypothesis, i.e., that the correlation coefficient is in fact zero. If this probability is lower than a conventional value, often taken to be 0.05 , the correlation coefficient is considered to be statistically significant.

Using a threshold of \pm 0.7 for a correlation or anticorrelation, respectively, we find only $2-3$ slice profiles out of 15 that fulfill this criterion. Changing the threshold to \pm 0.6 does not increase the number of possible correlated or anticorrelated slice profiles by more than 1 . Slice 4 has the most correlated profiles, and they are positively correlated. The next most correlated slices are 1 (negatively) and 2 (positively), followed by slices 5 (positively) and 10 (negatively). There is one more positively than negatively correlated slice, but this is not a statistically significant result.

We note that it is possible to obtain higher correlation numbers by excluding points at the ends of the slices. However, when doing so, we still do not find strong evidence of an anticorrelation (or correlation) anywhere else except at slice position 10.
Table 1

Correlation Coefficients Between the $8 \mu \mathrm{m}$ and Radio Continuum Emissions along the Slices Shown in Figure 9

\begin{tabular}{lccccc}
\hline \hline & \multicolumn{2}{c}{$\begin{array}{c}\text { Pearson } \\
\text { Correlation }\end{array}$} & & \multicolumn{2}{c}{$\begin{array}{c}\text { Spearman } \\
\text { Correlation }\end{array}$} \\
\cline { 2 - 3 } \cline { 5 - 6 } Slice & Coefficient & $P$-value & & Coefficient & $P$-value \\
\hline 1 & -0.68 & $4.3 \times 10^{-5}$ & & -0.75 & $2.3 \times 10^{-6}$ \\
2 & 0.88 & $1.2 \times 10^{-12}$ & & 0.75 & $1.1 \times 10^{-7}$ \\
3 & 0.41 & $1.5 \times 10^{-2}$ & & 0.49 & $2.8 \times 10^{-3}$ \\
4 & 0.89 & $1.7 \times 10^{-13}$ & & 0.91 & $9.9 \times 10^{-15}$ \\
5 & 0.55 & $3.8 \times 10^{-4}$ & & 0.61 & $7.2 \times 10^{-5}$ \\
6 & -0.22 & $1.8 \times 10^{-1}$ & & -0.27 & $1.0 \times 10^{-1}$ \\
7 & 0.22 & $2.0 \times 10^{-1}$ & & 0.25 & $1.4 \times 10^{-1}$ \\
8 & 0.25 & $6.8 \times 10^{-2}$ & & 0.41 & $2.5 \times 10^{-3}$ \\
9 & 0.16 & $3.0 \times 10^{-1}$ & & 0.08 & $6.1 \times 10^{-1}$ \\
10 & -0.58 & $7.4 \times 10^{-4}$ & & -0.60 & $4.2 \times 10^{-4}$ \\
11 & 0.17 & $1.9 \times 10^{-1}$ & & 0.22 & $9.0 \times 10^{-2}$ \\
12 & 0.32 & $4.0 \times 10^{-3}$ & & 0.35 & $1.8 \times 10^{-3}$ \\
13 & -0.27 & $3.7 \times 10^{-2}$ & & -0.08 & $5.4 \times 10^{-1}$ \\
14 & -0.32 & $1.8 \times 10^{-2}$ & & -0.44 & $6.8 \times 10^{-4}$ \\
15 & 0.30 & $1.0 \times 10^{-1}$ & & 0.35 & $5.4 \times 10^{-2}$ \\
\hline
\end{tabular}

We repeated this correlation analysis for the $8 \mu \mathrm{m}$ and $\mathrm{X}$-ray images and found no evidence of an anticorrelation (or correlation) between those two emissions. The only position where we see a significant anticorrelation is a little northwest of slice position 9 in Figure 8, where the radio continuum 

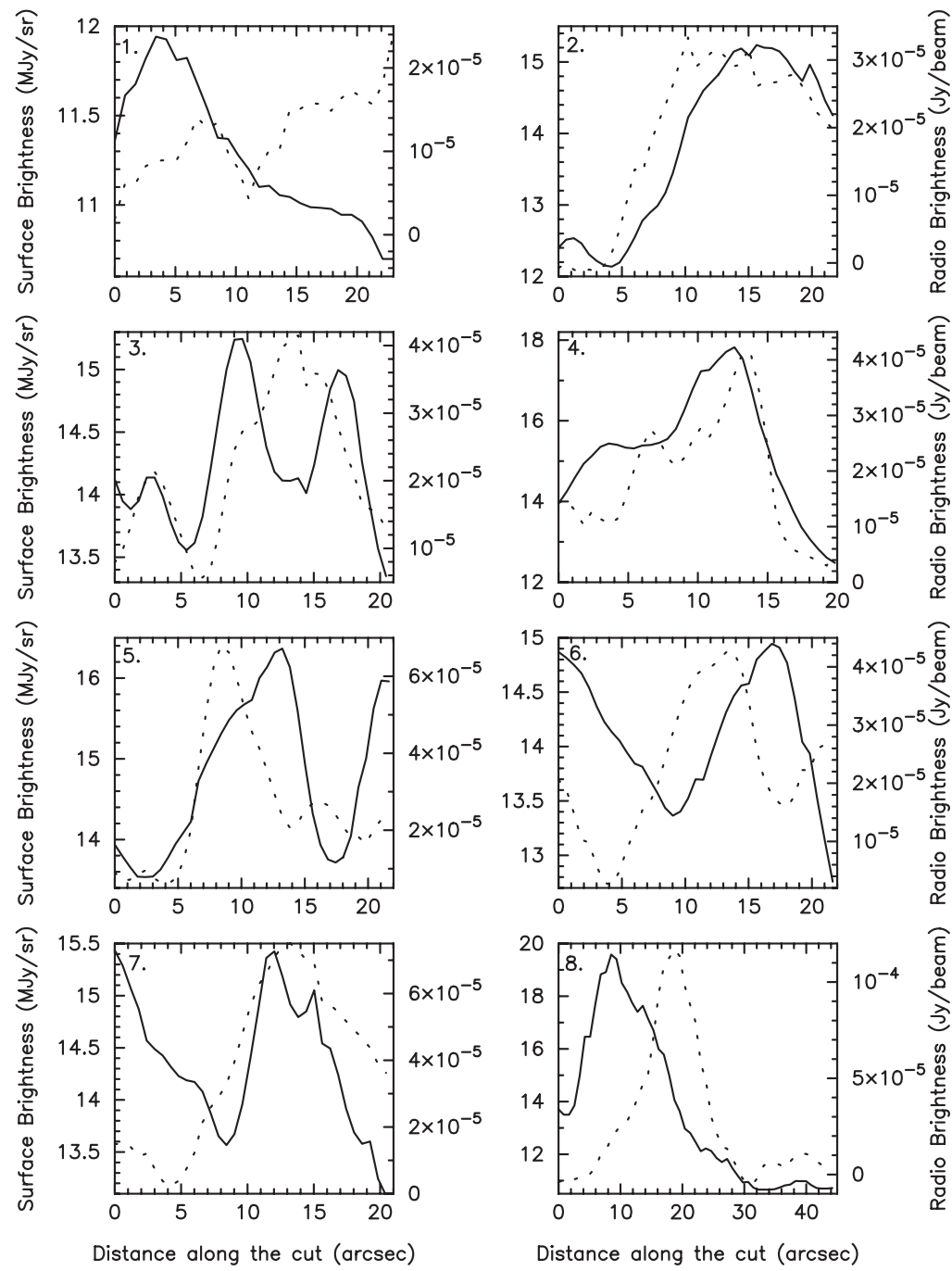

Figure 9. Slices taken across the radio jet as shown in Figure 8 in $8 \mu \mathrm{m}$ (solid line) and $3.6 \mathrm{~cm}$ radio emission (dashed line).

emission is relatively weak (but X-ray emission has a stronger ridge). However, this "anticorrelation" is due to the one-sided distribution (on the northeastern side only) of the $8 \mu \mathrm{m}$ emission with respect to the X-ray jet, and therefore does not provide evidence for any destruction of PAH molecules and dust in the jet path.

\section{DISCUSSION AND CONCLUSIONS}

Our data reveal that the $8 \mu \mathrm{m}$ emission (and the $5.8 \mu \mathrm{m}$ emission), believed to originate largely from PAH dust features and hot dust, is an excellent tracer of the normal spiral structure in NGC 4258, and hence it originates from the galactic plane. It allows us to distinguish between the normal spiral arms and the jet, which is not trivial, especially in the northern part. The other well-known spiral arm tracer, the $\mathrm{H} \alpha$ emission, cannot be used to distinguish the spiral arm and jet emissions in NGC 4258 as we find $\mathrm{H} \alpha$ emission (shock ionized) also along the inner jets.

We searched for a possible signature of dust destruction by the large-scale jets by comparing the $8 \mu \mathrm{m}$ emission to radio continuum emission in two different ways. A wavelet analysis shows that the $8 \mu \mathrm{m}$ emission exhibits a patchy structure, even along the large-scale jet, unlike the emission seen in the radio continuum (or $\mathrm{H} \alpha)$. All three studied emissions $(8 \mu \mathrm{m}, \mathrm{H} \alpha$, and radio continuum) show largest wavelet powers at around $30^{\prime \prime}(\sim 1 \mathrm{kpc})$ scales, mostly due to the central region. A correlation analysis of the wavelength-transformed emission at various scales shows that the $8 \mu \mathrm{m}$ emission is not correlated (or anticorrelated) with the radio continuum emission on any scale. Overall, the wavelet analysis shows that the $8 \mu \mathrm{m}$ emission traces the normal spiral arms much better than the radio continuum or $\mathrm{H} \alpha$ emissions.

We also took one-dimensional intensity profile cuts perpendicular to the radio continuum jet at several arbitrary positions along the jet. We only detected an anticorrelation between the $8 \mu \mathrm{m}$ and $3.6 \mathrm{~cm}$ radio continuum emissions far out in the disk where the jet has several branches. We consider this anticorrelation to be a fortuitous superposition of the two emissions rather than evidence for dust destruction. The profiles at smaller radii along the jet sometimes show a phase shift between the two emissions, but no systematic offset was found as a function of the distance from the nucleus or the side of the jet (north or south) with respect to the nucleus. We quantified these results by a thorough correlation coefficient analysis. Similar results (no correlation or anticorrelation) were obtained when comparing the $8 \mu \mathrm{m}$ and X-ray emissions.

In conclusion, we found that the large-scale jet speed in NGC 4258 (likely around $1000 \mathrm{~km} \mathrm{~s}^{-1}$; Cecil et al. 1992) is not sufficiently high to destroy dust traced by PAH emission on scales which are resolved by our observations. Our result is in 


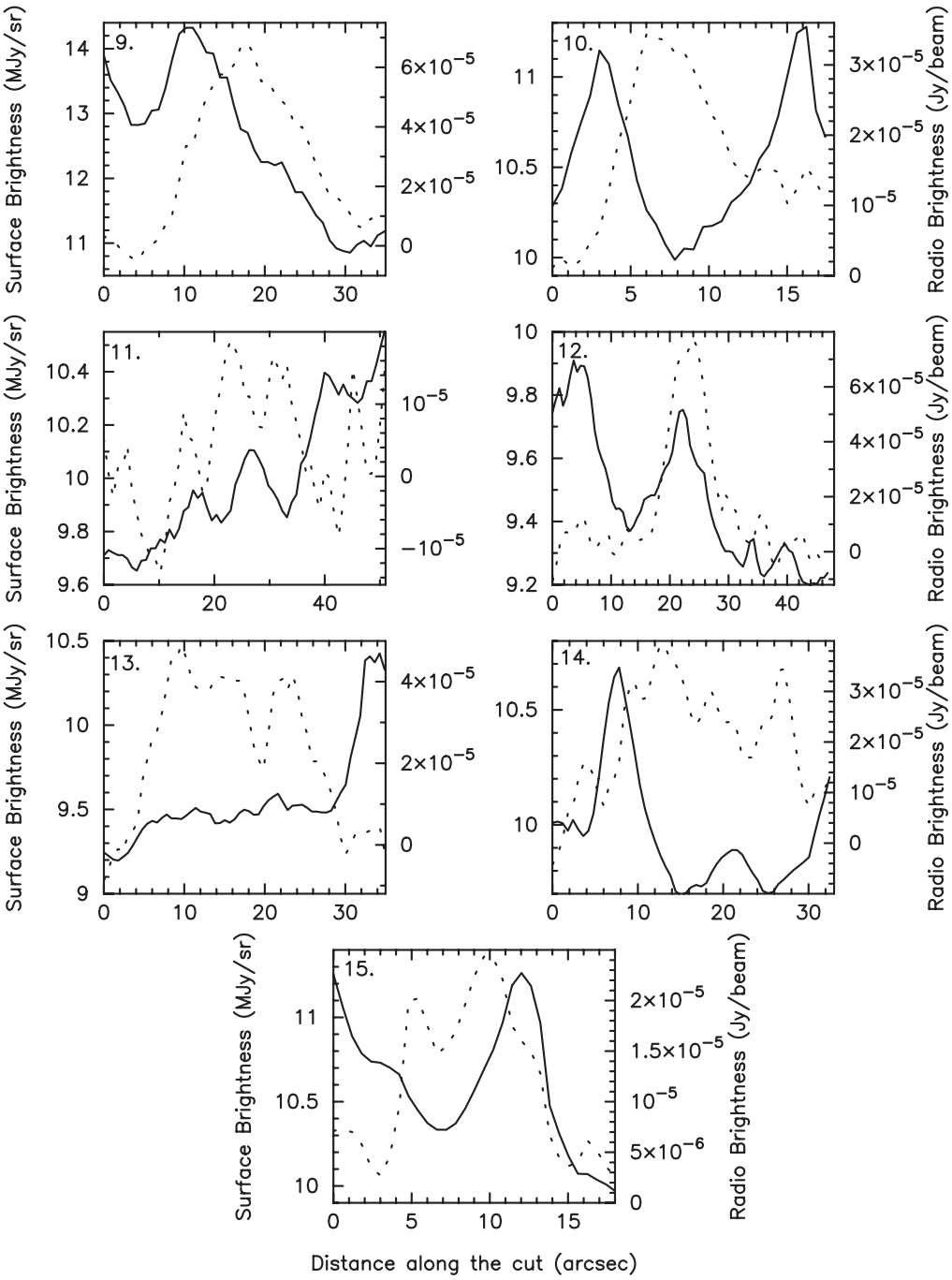

Figure 9. (Continued)

agreement with the conclusions of Dutil et al. (1995), who noted that dust destruction is not sufficient to explain the color of the jet of NGC 4258 in visible light. Future observations with higher resolution may detect dust destruction on smaller scales.

We thank Dr. Yuxuan Yang for sharing the reduced Chandra $\mathrm{X}$-ray data with us. We are grateful to Dr. Joe Hora for allowing us to use his custom muxbleed correction software. This work is based in part on observations made with the Spitzer Space Telescope, which is operated by the Jet Propulsion Laboratory, California Institute of Technology under a contract with NASA. Support for this work was provided by NASA through an award issued by JPL/Caltech.

Facilities: Spitzer(IRAC)

\section{REFERENCES}

Cecil, G., Morse, J. A., \& Veilleux, C. 1995, ApJ, 452, 613

Cecil, G., Wilson, A. S., \& Tully, R. B. 1992, ApJ, 390, 365

Cecil, G., et al. 2000, ApJ, 536, 675

Courtés, G., \& Cruvellier, P. 1961, C. R. Acad. Sci. Paris, 253, 218

Cox, P., \& Downes, D. 1996, ApJ, 473, 219

De Young, D. S. 1998, ApJ, 507, 161
Dutil, Y., Beauchamp, D., \& Roy, J.-R. 1995, ApJ, 444, L85

Fazio, G. G., et al. 2004, ApJS, 154, 10

Frick, P., Beck, R., Berkhuijsen, E. M., \& Patrickeyev, I. 2001, MNRAS, 327, 1145

Herrnstein, J. R., Greenhill, L. J., Moran, J. M., Diamond, P. J., Inoue, M., Nakai, N., \& Miyoshi, M. 1998, ApJ, 497, L69

Herrnstein, J. R., Moran, J. M., Greenhill, L. J., Diamond, P. J., Miyoshi, M., Nakai, N., \& Inoue, M. 1997, ApJ, 475, L17

Herrnstein, J. R., et al. 1999, Nature, 400, 539

Hummel, E., Krause, M., \& Lesch, H. 1989, A\&A, 211, 266

Krause, M., Cox, P., Garcia-Barreto, J. A., \& Downes, D. 1990, A\&A, 233, L1

Krause, M., Fendt, C., \& Neininger, N. 2007, A\&A, 467, 1037

Krause, M., \& Löhr, A. 2004, A\&A, 420, 115

Makovoz, D., \& Khan, I. 2005, in ASP Conf. Ser. 347, Astronomical Data Analysis Software and Systems VI, ed. P. L. Shopbell, M. C. Britton, \& R. Ebert (San Francisco, CA: ASP), 81

Miyoshi, M., Moran, J. M., Herrnstein, J. R., Greenhill, L., Nakai, N., Diamond, P., \& Inoue, M. 1995, Nature, 373, 127

Regan, M. W., et al. 2004, ApJS, 154, 204

Sawada-Satoh, S., Ho, P. T. P., Muller, S., Matsushita, S., \& Lim, J. 2007, ApJ, 658,851

Tabatabaei, F. S., et al. 2007, A\&A, 466, 509

van Albada, G. D. 1980, A\&A, 90, 123

van der Kruit, P. C., Oort, J. H., \& Mathewson, D. S. 1972, A\&A, 21, 169

Whittle, M. \& Wilson, A. S. 2004, AJ, 127, 606

Willner, S. P., et al. 2004, ApJS, 154, 222

Wilson, A. S., Yang, Y., \& Cecil, G. 2001, ApJ, 560, 689

Yang, Y., Li, B., Wilson, A. S., \& Reynolds, C. S. 2007, ApJ, 660, 1106 